Yukawa Institute for Theoretical Physics

YITP-13-15

Kyoto University

RUP-13-3

Department of Physics

Rikkyo University

\title{
Wave Effect in Gravitational Lensing by the Ellis Wormhole
}

\author{
Chul-Moon Yoo, ${ }^{1, *}$ Tomohiro Harada, ${ }^{2}$ and Naoki Tsukamoto ${ }^{2}$ \\ 1 Yukawa Institute for Theoretical Physics, \\ Kyoto University Kyoto 606-8502, Japan \\ ${ }^{2}$ Department of Physics, Rikkyo University, Tokyo 171-8501, Japan
}

We propose the use of modulated spectra of astronomical sources due to gravitational lensing to probe Ellis wormholes. The modulation factor due to gravitational lensing by the Ellis wormhole is calculated. Within the geometrical optics approximation, the normal point mass lens and the Ellis wormhole are indistinguishable unless we know the source's unlensed luminosity. This degeneracy is resolved with the significant wave effect in the low frequency domain if we take the deviation from the geometrical optics into account. We can roughly estimate the upper bound for the number density of Ellis wormholes as $n \lesssim 10^{-9} \mathrm{AU}^{-3}$ with throat radius $a \sim 1 \mathrm{~cm}$ from the existing femto-lensing analysis for compact objects.

*Electronic address: yoo@yukawa.kyoto-u.ac.jp 


\section{INTRODUCTION}

In a variety of cosmological models based on fundamental theory, exotic astrophysical objects which have not been observed are often predicted. Conversely, an observational evidence for an exotic object would stimulate creative theoretical discussions. Probing these exotic objects and detecting them will give us significant progress of research in fundamental physics. Even if we cannot detect it, giving a constraint on the abundance of the exotic objects is one of powerful means of investigating the nature of our universe.

Generally, the interaction between such unobserved exotic objects and well known matters is very weak or not well established. Thus, only the gravitational interaction would cause reliable observational phenomena. One of the most direct measurements of gravitational effects of an exotic object is gravitational lensing. For instance, massive compact halo objects are probed by using micro-lensing 1 [3]. Cosmic strings are also targets for probing by using gravitational lensing phenomena [4 -12]. In this paper, we propose a way to probe Ellis wormholes [13] by using lensed spectra of astronomical sources.

The Ellis wormhole was first introduced by Ellis as a spherically symmetric solution of Einstein equations with a ghost massless scalar field. The dynamical stability of the Ellis wormhole is discussed in Ref. [14] and the possible source to support the Ellis geometry was proposed in Ref. [15]. Gravitational lensing by the Ellis wormhole was studied in Refs. [16, 17] and recently revisited by several authors [18, 19]. So far, it has been suggested that Ellis wormholes can be probed by using light curves of gamma-ray bursts [20], microlensing [21 23] (see also Refs. [24, 25]) and imaging observations [26, 27], while our proposal is the use of spectroscopic observations to probe Ellis wormholes.

In order to fully investigate the lensed spectrum of a point source, the wave effect in gravitational lensing must be taken into account. The wave effect for the point mass lens is discussed in Refs. [28, 29]. Wave effects in gravitational lensing by the rotating massive object [30], binary system [31], singular isothermal sphere [32] and the cosmic string [33, 34] have been considered. In Sec. III, we calculate the amplification factor of gravitational lensing by the Ellis wormhole taking the wave effect into account. The geometrical optics limit is analytically presented in Sec. III. The difference in the amplification factor between the point mass lens and the Ellis wormhole lens is discussed in Sec. IV based on observables. In Sec. Q possible observations to probe Ellis wormholes are listed. Sec. VI is devoted to a summary.

In this paper, we use the geometrized units in which the speed of light and Newton's gravitational constant are both unity.

\section{A DERIVATION OF THE LENSED WAVE FORM}

The line element in the Ellis wormhole spacetime can be written by the following isotropic form:

$$
\mathrm{d} s^{2}=-\mathrm{d} t^{2}+\left(1+\frac{a^{2}}{R^{2}}\right)^{2}\left(\mathrm{~d} R^{2}+R^{2} \mathrm{~d} \Omega^{2}\right),
$$


where $R=a$ corresponds to the throat and we simply call $a$ the throat radius in this paper. ${ }^{1}$ Assuming the thin lens approximation is valid, we consider the wormhole lens system shown in Fig. 1. We use the position vector $\vec{X}=(X, Y, Z)$ in the flat space. Then, the

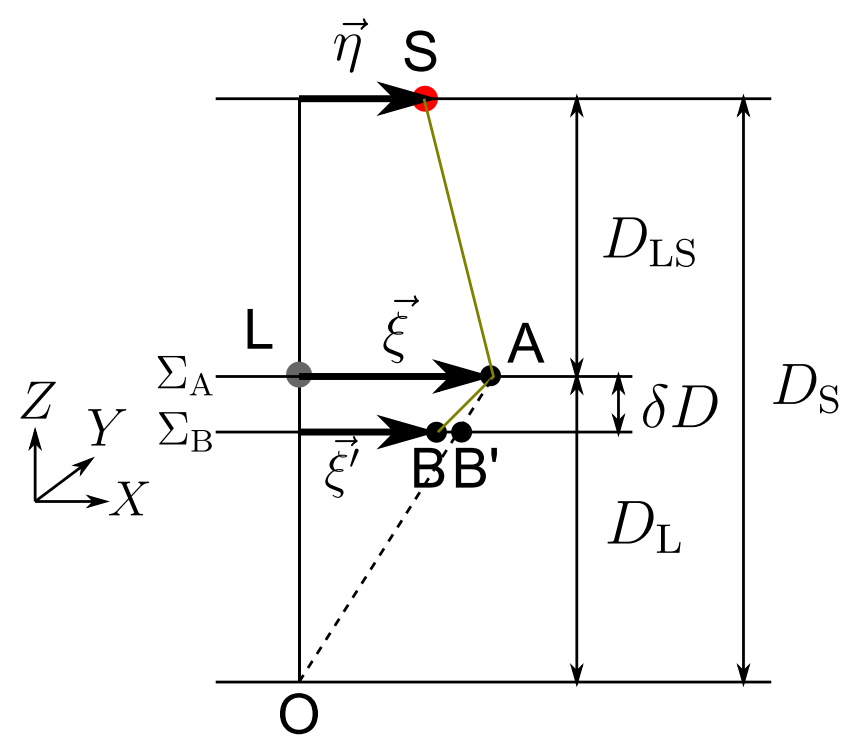

FIG. 1: Lens system with thin lens approximation. S, L, and O represent the source, lens, and observer positions, respectively. The path SAB is a ray trajectory which is specified with the vector $\vec{\xi}$ on the lens plane $\Sigma_{\mathrm{A}} \cdot \mathrm{B}^{\prime}$ is the intersection of the line $\mathrm{AO}$ and the plane $\Sigma_{\mathrm{B}} \cdot \vec{\xi}^{\prime}$ is the position vector of the point $\mathrm{B}$ on the plane $\Sigma_{\mathrm{B}}$.

coordinate $R$ is given by $R=\left|\vec{X}-\vec{X}_{\mathrm{L}}\right|$, where $\left|\vec{X}_{\mathrm{L}}\right|$ is the lens position. We set $Z$-axis as the perpendicular direction to the lens plane and the source plane. $D_{\mathrm{S}}, D_{\mathrm{L}}$, and $D_{\mathrm{LS}}$ denote the distances from the observer plane to the source plane, from the observer plane to the lens plane, and from the lens plane to the source plane, respectively.

In the geometrical optics limit, we consider light rays emanated from the source. The vector $\vec{\xi}$ on the lens plane $\Sigma_{\mathrm{A}}$ in Fig. 1 specifies the light ray which is deflected once at $\vec{X}=\vec{X}_{\mathrm{L}}+\vec{\xi}$. Since $\xi:=|\vec{\xi}|$ can be regarded as the closest approach of the light ray, as is shown in Ref. [16], the deflection angle $\alpha$ is given by

$$
\alpha(\xi)=\pi\left(\frac{a}{\xi}\right)^{2}+\mathcal{O}\left(\frac{a}{\xi}\right)^{4} .
$$

As a result, the Einstein radius $\xi_{0}$ for the Ellis wormhole is given by

$$
\xi_{0}=\left(\frac{1}{4} \pi a^{2} D\right)^{1 / 3}
$$

\footnotetext{
${ }^{1}$ Since the throat surface area is given by $16 \pi a^{2}$, our definition of the throat radius is half the areal radius of the throat.
} 
where

$$
D=\frac{4 D_{\mathrm{L}} D_{\mathrm{LS}}}{D_{\mathrm{S}}}
$$

Since we are interested in the wave effect, that is, the deviation from the geometrical optics limit, we need to treat the wave equation rather than light rays. Neglecting the polarization effect, we consider the scalar wave equation with the frequency $\omega$. The wave equation for the monochromatic wave $\mathrm{e}^{i \omega t} \phi(\vec{X})$ is given by

$$
\omega^{2} \phi+\left(1+\frac{a^{2}}{R^{2}}\right)^{-3} \partial_{i}\left[\left(1+\frac{a^{2}}{R^{2}}\right) \delta^{i j} \partial_{j} \phi\right]=-4 \pi A \delta\left(\vec{X}-\vec{X}_{\mathrm{S}}\right)
$$

where $\vec{X}_{\mathrm{S}}$ is the position vector of the point source and $A$ in the source term is a constant which specifies the amplitude. Without the wormhole, we obtain the wave form $\bar{\phi}_{\mathrm{O}}$ at the observer O as follows:

$$
\bar{\phi}_{\mathrm{O}}=\frac{A}{\sqrt{D_{\mathrm{S}}^{2}+\eta^{2}}} \exp \left[i \omega \sqrt{D_{\mathrm{S}}^{2}+\eta^{2}}\right]=\frac{A}{D_{\mathrm{S}}} \exp \left[i \omega D_{\mathrm{S}}\left(1+\frac{\eta^{2}}{2 D_{\mathrm{S}}^{2}}+\mathcal{O}\left(\left(\frac{\eta}{D_{\mathrm{S}}}\right)^{4}\right)\right)\right]
$$

where $\eta=|\vec{\eta}|$ and $\vec{\eta}=\left(X_{\mathrm{S}}-X_{\mathrm{L}}, Y_{\mathrm{S}}-Y_{\mathrm{L}}, 0\right)$ and we consider the case $\eta \ll D_{\mathrm{S}}$ in this paper.

Our assumptions to calculate the wave form at $\mathrm{O}$ are summarized as follows(see Ref. [29]):

(a) The geometrical optics approximation is valid between the source plane and the plane $\Sigma_{\mathrm{B}}$ in Fig. 1.

(b) Thin lens approximation is valid and a ray from the source is deflected once on the lens plane $\Sigma_{\mathrm{A}}$.

(c) Assuming $D_{\mathrm{S}} \sim D_{\mathrm{L}} \sim D_{\mathrm{LS}} \sim D$, we use a non-dimensional parameter $\epsilon$ defined by $\epsilon:=\xi_{0} / D$, which gives the typical scale of the deflection angle. Then, we assume $1 /(\omega D) \ll \epsilon \ll 1$ and $\eta / D=\mathcal{O}(\epsilon)$.

(d) On the plane $\Sigma_{\mathrm{B}}$, the gravitational potential of the lens object is negligible and $\delta D / D=\mathcal{O}(\epsilon)$, where $\delta D$ is the distance between the planes $\Sigma_{\mathrm{A}}$ and $\Sigma_{\mathrm{B}}$.

On the assumptions made above, we calculate the wave form on the plane $\Sigma_{\mathrm{B}}$ up to the leading order for the amplitude and next leading terms for the phase part. Applying the Kirchhoff integral theorem between the plane $\Sigma_{\mathrm{B}}$ and the observer, we calculate the approximate wave form at $\mathrm{O}$.

The vector $\vec{\xi}$ specifies the light ray which is deflected once on the plane $\Sigma_{\mathrm{A}}$ at $\vec{X}=\vec{X}_{\mathrm{L}}+\vec{\xi}$ and reaches the plane $\Sigma_{\mathrm{B}}$. The deflection angle is fixed by $\vec{\xi}$ and the background geometry. The point A in Fig. 1 denotes the deflected point. We label the intersection of the deflected light ray and the plane $\Sigma_{\mathrm{B}}$ as $\mathrm{B}$, while $\mathrm{B}^{\prime}$ in the Fig. 1 denotes the intersection of the line AO and the plane $\Sigma_{\mathrm{B}}$. As will be mentioned at the end of this section, the dominant contribution to the wave form at $\mathrm{O}$ comes from rays which satisfy $\xi \sim \epsilon D$, where $\xi=|\vec{\xi}|$. Therefore we consider $\vec{\xi} / D$ as $\mathcal{O}(\epsilon)$ hereafter. 
First, we consider the following ansatz for $\phi$ in the region between the source plane and the plane $\Sigma_{\mathrm{B}}$ :

$$
\phi=f(\vec{X}) \mathrm{e}^{i S(\vec{X})} .
$$

On the plane $\Sigma_{\mathrm{B}}$, the amplitude $f(\vec{X})$ is given by

$$
\left.f(\vec{X})\right|_{\Sigma_{\mathrm{B}}}=\frac{A}{D_{\mathrm{LS}}}(1+\mathcal{O}(\epsilon))
$$

In the geometrical optics approximation, the phase $S(\vec{X})$ satisfies the eikonal equation given by

$$
\delta^{i j} \partial_{i} S \partial_{j} S=\omega^{2}\left(1+\frac{a^{2}}{R^{2}}\right)^{2} .
$$

At the point $\mathrm{B}$, the phase based on the source position $\mathrm{S}$ is given by the following integral

$$
\left.S\right|_{\mathrm{B}}=\int_{\mathrm{S}}^{\mathrm{B}} \frac{\mathrm{d} x^{i}}{\mathrm{~d} l} \partial_{i} S d l
$$

where we have introduced the optical path length $l$ defined as

$$
\delta_{i j} \frac{\mathrm{d} x^{i}}{\mathrm{~d} l} \frac{\mathrm{d} x^{j}}{\mathrm{~d} l}=1
$$

Since, in the geometrical optics approximation, we find

$$
\partial_{j} S=\omega\left(1+\frac{a^{2}}{R^{2}}\right) \delta_{i j} \frac{\mathrm{d} x^{i}}{\mathrm{~d} l}
$$

the integral (10) is given by

$$
\left.S\right|_{\mathrm{B}}=\omega \int_{\mathrm{S}}^{\mathrm{B}} d l+\omega a^{2} \int_{\mathrm{S}}^{\mathrm{B}} \frac{1}{R^{2}} d l .
$$

After the calculations explicitly shown in Appendix A, we finally obtain the following expression:

$$
\left.S\right|_{B} \simeq \omega\left[D_{\mathrm{S}}\left(1+\frac{\eta^{2}}{2 D_{\mathrm{S}}^{2}}\right)+\frac{D_{\mathrm{L}} D_{\mathrm{S}}}{2 D_{\mathrm{LS}}}\left(\frac{\vec{\xi}}{D_{\mathrm{L}}}-\frac{\vec{\eta}}{D_{\mathrm{S}}}\right)^{2}-r+\frac{\pi a^{2}}{\xi}\right]
$$

This expression for the phase and Eq. (8) for the amplitude can be used for any value of $\vec{\xi}$, that is, we have obtained an approximate wave form on the plane $\Sigma_{\mathrm{B}}$.

Applying the Kirchhoff integral theorem[35] and neglecting the contribution from the infinity, we express the wave form $\phi_{\mathrm{O}}$ at $\mathrm{O}$ by the following integral:

$$
\phi_{\mathrm{O}}=-\frac{1}{4 \pi} \int_{\Sigma_{\mathrm{B}}} \mathrm{d} \xi^{2}\left\{\phi_{\mathrm{B}} \frac{\partial}{\partial Z}\left(\frac{\mathrm{e}^{i \omega r}}{r}\right)-\frac{\mathrm{e}^{i \omega r}}{r} \frac{\partial \phi_{\mathrm{B}}}{\partial Z}\right\},
$$


where $\phi_{\mathrm{B}}$ is the waveform at B. Since we are interested in only the leading order of the amplitude, we obtain

$\phi_{\mathrm{O}} \simeq-\frac{i \omega A}{2 \pi D_{\mathrm{L}} D_{\mathrm{LS}}} \exp \left[i \omega D_{\mathrm{S}}\left(1+\frac{\eta^{2}}{2 D_{\mathrm{S}}^{2}}\right)\right] \int \mathrm{d} \xi^{2} \exp \left[i \omega\left\{+\frac{D_{\mathrm{L}} D_{\mathrm{S}}}{2 D_{\mathrm{LS}}}\left(\frac{\vec{\xi}}{D_{\mathrm{L}}}-\frac{\vec{\eta}}{D_{\mathrm{S}}}\right)^{2}+\frac{\pi a^{2}}{\xi}\right\}\right]$,

where we have used the following approximations:

$$
\begin{aligned}
& \frac{\partial}{\partial Z}\left(\frac{\mathrm{e}^{i \omega r}}{r}\right) \simeq \frac{i \omega}{r} \mathrm{e}^{i \omega r} \simeq \frac{i \omega}{D_{\mathrm{L}}} \mathrm{e}^{i \omega r}, \\
& \frac{\partial \phi_{\mathrm{B}}}{\partial Z} \simeq \frac{-i \omega A}{D_{\mathrm{LS}}} \mathrm{e}^{\left.i S\right|_{B}} .
\end{aligned}
$$

Defining the amplification factor $F$ by $F:=\phi_{\mathrm{O}} / \bar{\phi}_{\mathrm{O}}$, we obtain

$$
F \simeq \frac{\omega d}{\pi i} \int \mathrm{d} x^{2} \exp \left[i \omega d\left\{(\vec{x}-\vec{y})^{2}+\frac{2}{x}\right\}\right]
$$

where

$$
\begin{aligned}
d & =\frac{\xi_{0}^{2} D_{\mathrm{S}}}{2 D_{\mathrm{LS}} D_{\mathrm{L}}}=\frac{2 \xi_{0}^{2}}{D}=2 \xi_{0} \epsilon, \\
\vec{x} & =\frac{\vec{\xi}}{\xi_{0}} \\
\vec{y} & =\frac{\vec{\eta} D_{\mathrm{L}}}{\xi_{0} D_{\mathrm{S}}}
\end{aligned}
$$

$d$ gives the optical path difference between the lensed trajectory and the unlensed one in the geometrical optics limit for $\vec{\eta}=0$.

Introducing a polar coordinate, we rewrite this integral as

$$
\begin{aligned}
F & \simeq \frac{\omega d}{\pi i} \mathrm{e}^{i \omega d y^{2}} \int_{0}^{\infty} \mathrm{d} x x \exp \left[i \omega d\left(x^{2}+\frac{2}{x}\right)\right] \int_{0}^{2 \pi} \mathrm{d} \varphi \exp [-2 i \omega d x y \cos \varphi] \\
& =-2 i \omega d \mathrm{e}^{i \omega d y^{2}} \int_{0}^{\infty} \mathrm{d} x x \exp \left[i \omega d\left(x^{2}+\frac{2}{x}\right)\right] J_{0}(2 \omega d x y) .
\end{aligned}
$$

The integrand is divergent at the infinity on the real axis. This is caused by our approximation associated with $\epsilon$, and not real. If we write down the integrand in a precise form without any approximation, we do not have any divergence. Actually, in the precise form, the contribution from the integral in the region $x \gg 1$ is negligible due to the cancellation of the quasi-periodic integration. For the same reason, the contribution from the integration in the region $x \ll 1$ is also negligible. Since the approximate expression of Eq. (24) is valid in the region $x \ll 1 / \epsilon$, we can obtain a reliable result by neglecting the contribution from the region $x \gg 1$ in the integral (24). Practically, to make this integral finite, it is convenient to consider the analytic continuation to the complex plane and take the path of the integral as shown in Fig. 2, Then, this integral can be numerically performed. 


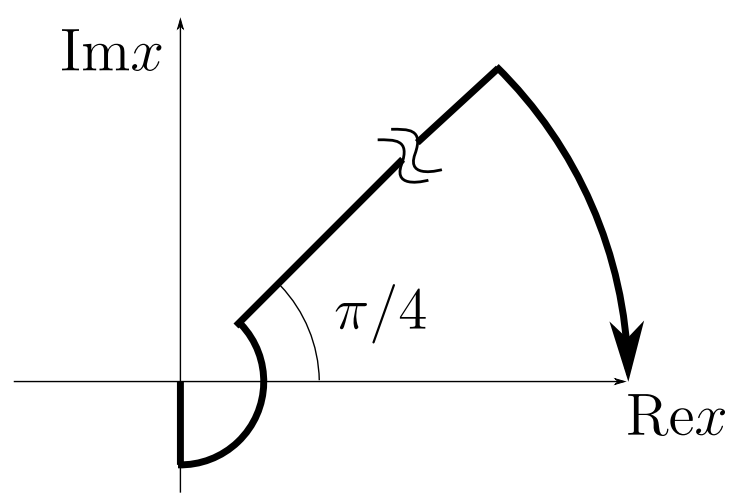

FIG. 2: The path of the integral (24) taken in the numerical integration.

\section{GEOMETRICAL OPTICS APPROXIMATION}

In this section, we derive an approximate form of the amplification factor $F$. In the expression (23), we apply the stationary phase approximation to the integral with respect to $x$. Then we obtain

$$
F \simeq \sqrt{\frac{\omega d}{\pi i}} \int_{0}^{2 \pi} \mathrm{d} \varphi \frac{x_{0}}{\sqrt{1+2 / x_{0}^{3}}} \exp \left[i \omega d h\left(x_{0}\right)\right],
$$

where

$$
h(x)=x^{2}-2 x y \cos \varphi+\frac{2}{x}
$$

and $x_{0}=x_{0}(\varphi)>0$ satisfies

$$
h^{\prime}\left(x_{0}\right)=0 \Leftrightarrow x_{0}^{3}-x_{0}^{2} y \cos \varphi-1=0 .
$$

Note that Eq. (27) has only one positive root as a function of $\varphi$.

In Eq. (25), we again perform the stationary phase approximation in the integral with respect to $\varphi$, and we obtain

$$
\begin{aligned}
F \simeq F_{\text {geo }}= & \left(\frac{x_{+}^{3}}{\sqrt{\left(x_{+}^{3}+2\right)\left(x_{+}^{3}-1\right)}} \exp \left[i \omega d \frac{-x_{+}^{3}+4}{x_{+}}\right]\right. \\
& \left.+\frac{x_{-}^{3}}{\sqrt{\left(x_{-}^{3}+2\right)\left(1-x_{-}^{3}\right)}} \exp \left[i \omega d \frac{-x_{-}^{3}+4}{x_{-}}-\frac{i \pi}{2}\right]\right),
\end{aligned}
$$

where $x_{ \pm}$satisfies

$$
x_{ \pm}^{3} \mp x_{ \pm}^{2} y-1=0 .
$$

Note that $1<x_{+}$and $0<x_{-}<1$. If we define $\mu_{ \pm}$and $\theta_{ \pm}$as

$$
\begin{aligned}
\mu_{ \pm} & =\frac{x_{ \pm}^{6}}{\left(x_{ \pm}^{3}+2\right)\left(x_{ \pm}^{3}-1\right)} \\
\theta_{ \pm} & =\omega d \frac{-x_{ \pm}^{3}+4}{x_{ \pm}}-\frac{\pi}{4} \pm \frac{\pi}{4}
\end{aligned}
$$


$F_{\text {geo }}$ can be expressed as

$$
F_{\text {geo }}=\sum_{ \pm} \sqrt{\left|\mu_{ \pm}\right|} e^{i \theta_{ \pm}}
$$

$\mu_{ \pm}$is the magnification factor for each image in the geometrical optics approximation.

As an observable, we focus on $|F|^{2}$ in this paper. In the geometrical optics approximation, we obtain

$$
\left|F_{\text {geo }}\right|^{2}=\left|\mu_{+}\right|+\left|\mu_{-}\right|+2 \sqrt{\left|\mu_{+} \mu_{-}\right|} \sin (2 \omega d \tau(y))
$$

with $\tau(y)$ being the following:

$$
\tau(y):=\frac{\theta_{-}-\theta_{+}+\pi / 2}{2 \omega d},
$$

where note that the definition of $\tau$ can be written in terms of $x_{ \pm}$, which is a function of $y$. $|F|^{2}$ and $\left|F_{\text {geo }}\right|^{2}$ are depicted as functions of $\omega$ for each value of $y$ in Fig. 3 .
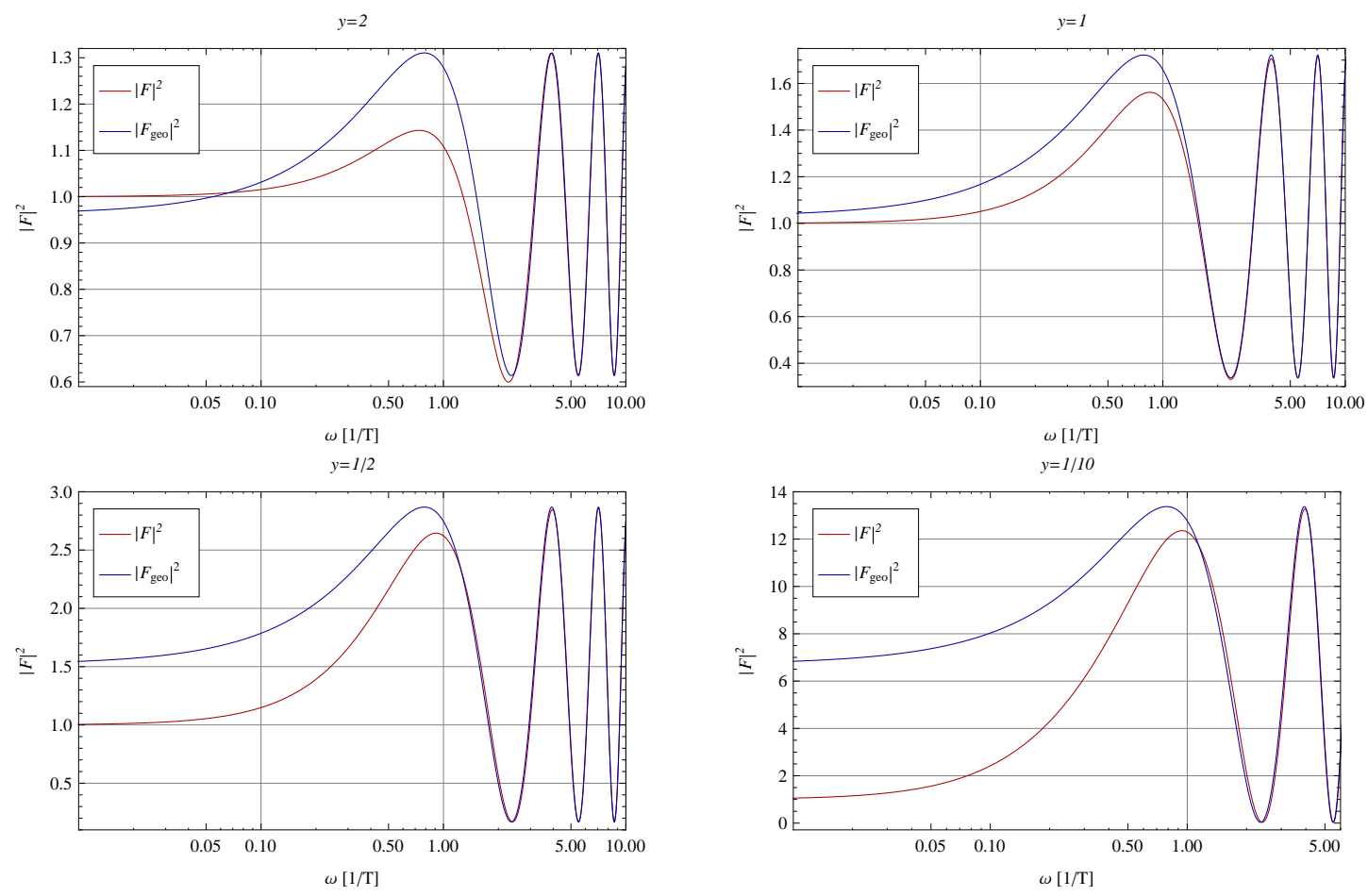

FIG. 3: $|F|^{2}$ and $\left|F_{\text {geo }}\right|^{2}$ for the wormhole lens, where $T=d \tau$.

\section{COMPARISON WITH THE POINT MASS LENS BASED ON OBSERVABLES}

In this paper, we assume the following situations for the observation:

- We can observe the spectrum of a source. 
- The unlensed spectrum shape is well known.

Note that, in our analysis, knowledge about the luminosity is not necessary. The amplification factor for the point mass lens is summarized in Appendix B. For both point mass and wormhole cases, in the geometrical optics approximation, we obtain the form of Eq. (33) or equivalently Eq. (B3).

In the frequency region where the geometrical optics approximation is valid, there are basically three observables which characterize the form of the amplification factor. The first is the frequency $\omega$, the second is the period of the oscillation of the spectrum as a function of $\omega$, and the third is the ratio $\kappa$ between the amplitude of the oscillation and the mean value. The period of the oscillation of the spectrum makes $T:=\tau(y) d$ an observable. $\kappa$ is given by

$$
\kappa=\frac{2 \sqrt{\left|\mu_{+} \mu_{-}\right|}}{\left|\mu_{+}\right|+\left|\mu_{-}\right|}
$$

from Eq. (33) and plotted as a function of $y$ as is shown in Fig. (4. Since $\kappa$ is observable, $y$

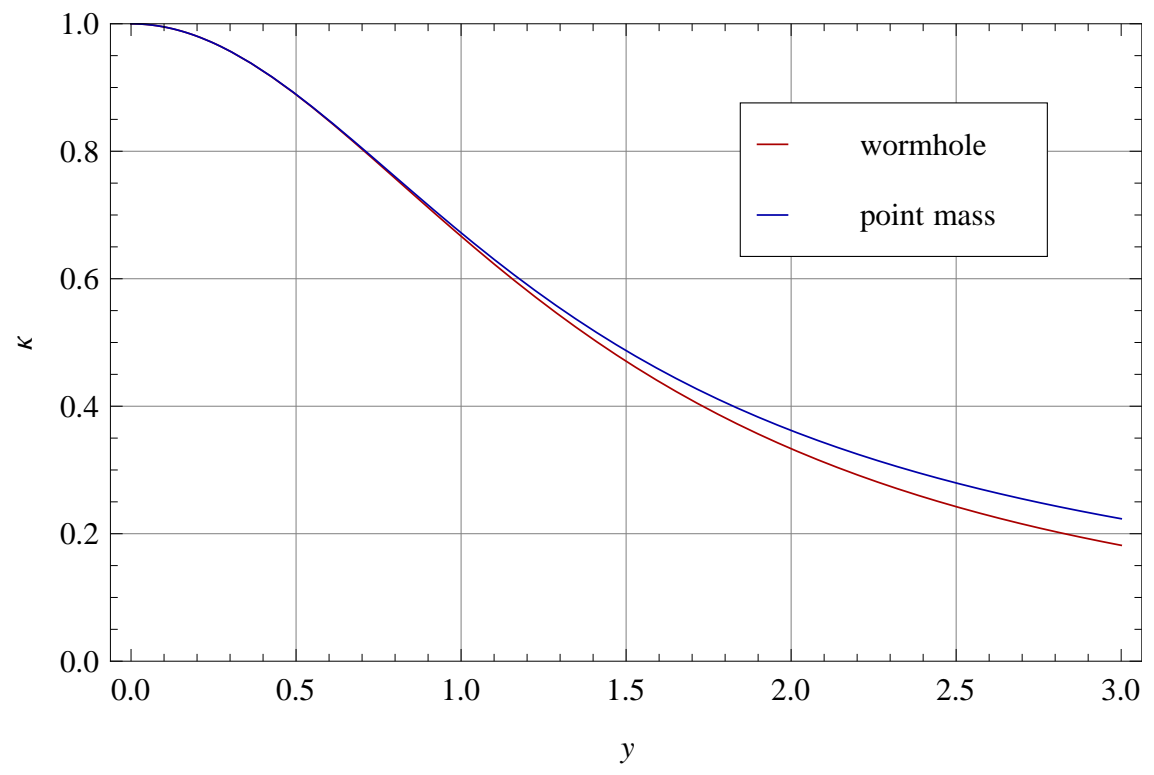

FIG. 4: $\kappa$ as a function of $y$ for the Ellis wormhole case and the point mass lens case.

and hence $\tau(y)$ can be determined if we have enough accuracy of the observation. As shown in Fig. 5. $\tau(y)$ is a monotonically increasing function of $y$ and close to $2 y$ in the region $y<1$. Then, from another observable $T=\tau(y) d$ we can obtain the value of $d$.

The situation for the point mass lens case is the same as for the Ellis wormhole case. That is, the three observables $\omega, T$, and $\kappa$ can be regarded as gravitational lensing by a point mass as well as a wormhole. This fact indicates that we cannot distinguish which is the lens object only by using these three observables in the geometrical optics approximation. This degeneracy is resolved in the small frequency region in which the wave effect becomes significant as is explicitly shown in Fig. 6. 


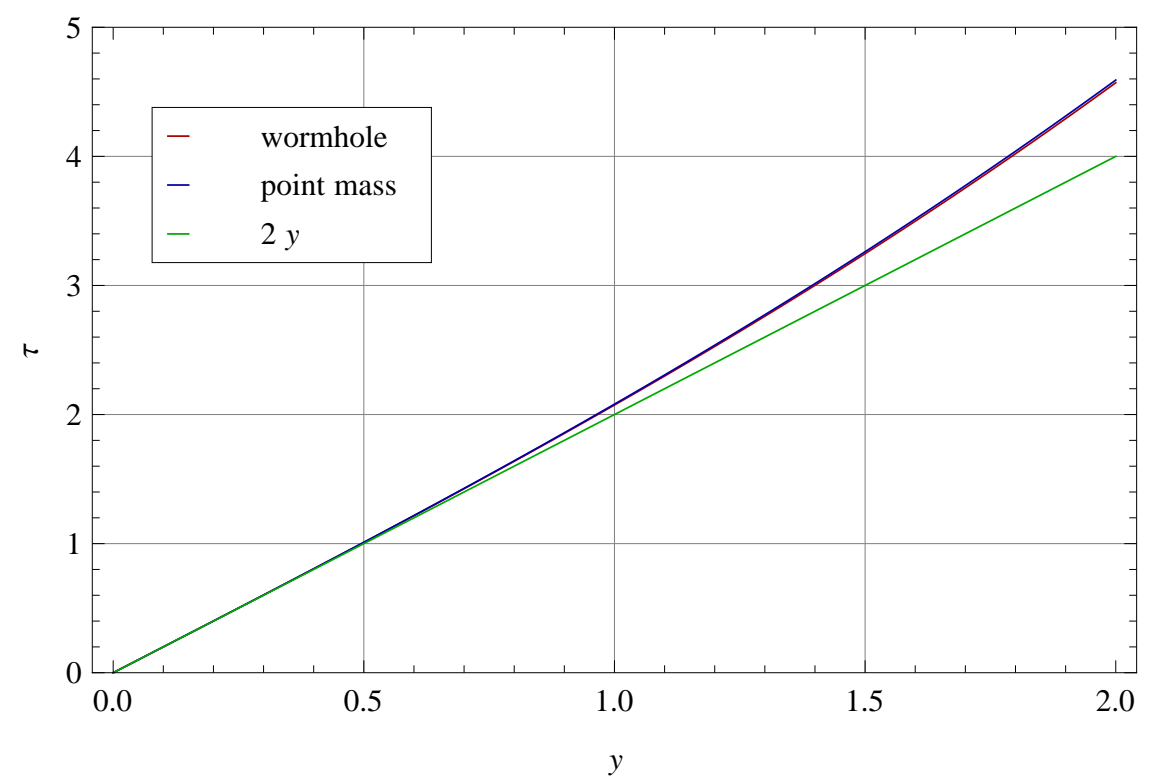

FIG. 5: $\tau(y)$ for the Ellis wormhole case and the point mass lens case. The two cases are almost indistinguishable from each other in the region depicted here.
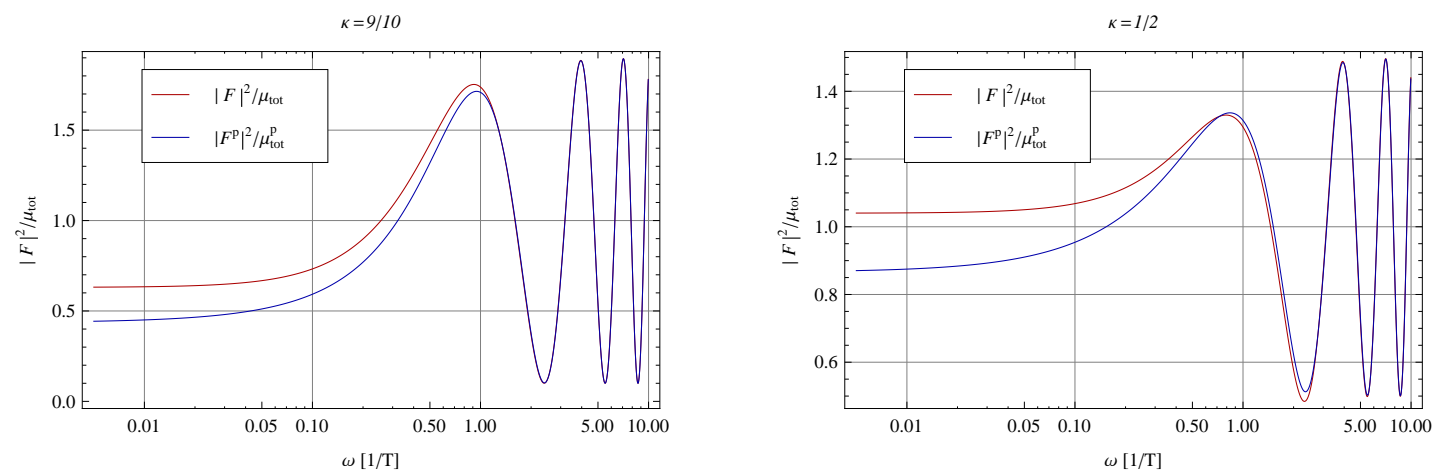

FIG. 6: Comparison between $|F|^{2}$ and $\left|F^{\mathrm{p}}\right|^{2}$.

To simply see this resolution of the degeneracy, we consider the small frequency limit, i.e., $\omega \rightarrow 0$. In this limit, we have $F \rightarrow 1$ as explicitly shown in Figs. 3 and 8 , If we can observe the spectrum in any frequency region of interest, the following quantity is an observable:

$$
\frac{\lim _{\omega \rightarrow 0}|F|^{2}}{\lim _{\omega \rightarrow \infty}<|F|^{2}>}=1 / \mu_{\text {tot }}:=1 /\left(\left|\mu_{+}\right|+\left|\mu_{-}\right|\right),
$$

where the bracket $<>$ denotes the average through several periods. We depict $1 / \mu_{\text {tot }}$ as a function of $y$ for both the wormhole case and the point mass lens case in Fig. 7. In both cases, $1 / \mu_{\text {tot }}$ approaches to 0 and 1 in the limits $y \rightarrow 0$ and $y \rightarrow \infty$, respectively. While $1 / \mu_{\text {tot }}<1$ is satisfied in all domain of $y$ for the point mass lens, $1 / \mu_{\text {tot }}$ can exceed 1 for the wormhole case due to the demagnification effect originated from the negative mass density 


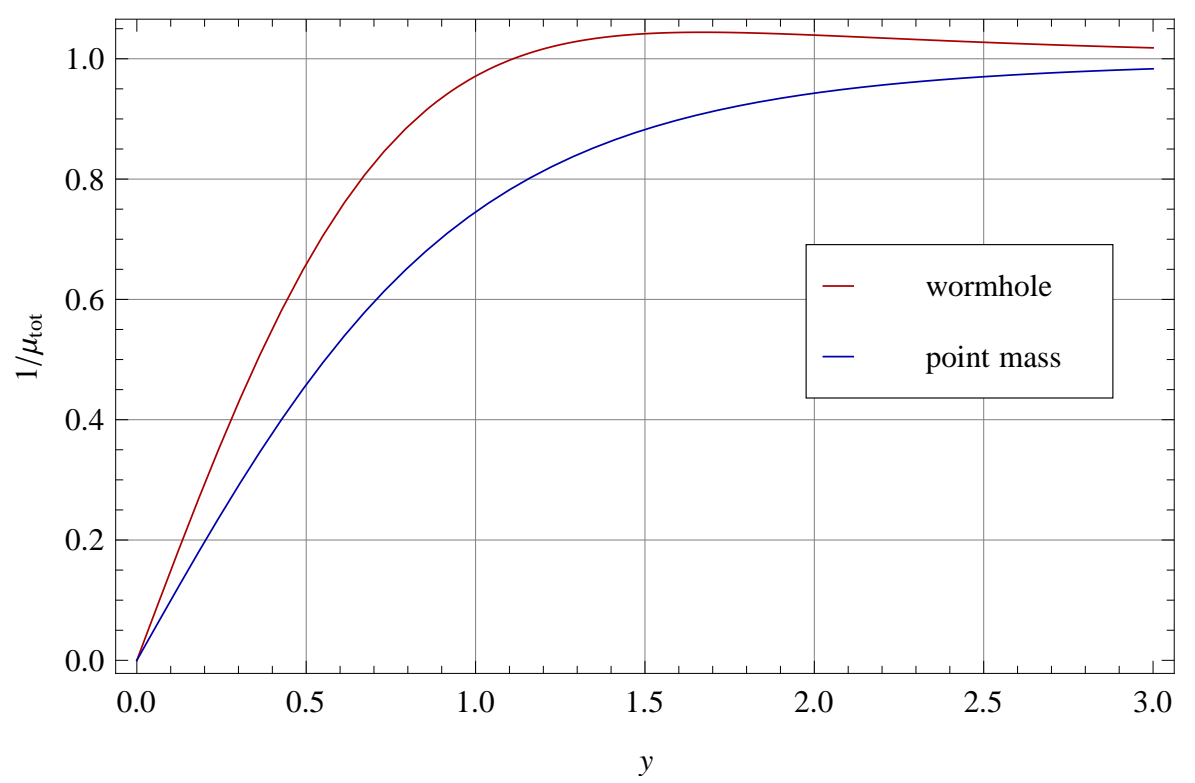

FIG. $7: 1 / \mu_{\text {tot }}$

surrounding the Ellis wormhole. The behaviours of $1 / \mu_{\text {tot }}$ are totally different from each other. This fact shows that they are, in principle, distinguishable.

\section{OBSERVATIONAL CONSTRAINT}

One of the possible sources is gamma-ray bursts, which have been proposed to be used for probing small mass primordial black holes [36 39] and low tension cosmic strings 34]. Recently, the femto-lensing effects caused by compact objects were searched by using gammaray bursts with known redshifts detected by the Fermi Gamma-ray Burst Monitor [40]. From non-detection of the femto-lensing event, a constraint on the number density of compact objects have been obtained. For a fixed value of the mass $M$ of each compact object, the constraint can be translated into a constraint on $\Omega_{\mathrm{CO}}$, where $\Omega_{\mathrm{CO}}$ is the average energy density of compact objects in the unit of the critical density $\rho_{\mathrm{cr}}$. Then, the abundance of dark compact objects is constrained as $\Omega_{\mathrm{CO}}<0.15$ at the $95 \%$ confidence level for $M \sim 3 \times 10^{18} \mathrm{~g}$.

Since the number density $n$ of the compact objects is given by

$$
n=\frac{\Omega_{\mathrm{CO}} \rho_{\mathrm{cr}}}{M} \sim 2 \times 10^{-9} \mathrm{AU}^{-3}\left(\frac{\Omega_{\mathrm{CO}}}{0.15}\right)\left(\frac{M}{3 \times 10^{18} \mathrm{~g}}\right)^{-1}\left(\frac{\rho_{\mathrm{cr}}}{10^{-29} \mathrm{~g} / \mathrm{cm}^{3}}\right),
$$

we obtain the constraint for the number dnsity of compact objects with $M \sim 3 \times 10^{18} \mathrm{~g}$ as $n<2 \times 10^{-9} \mathrm{AU}^{-3}$. Then, we can expect a similar constraint on the number density of Ellis wormholes with the throat radius which gives the same value of $d$ as that for the compact object. The mass of $3 \times 10^{18} \mathrm{~g}$ gives $d \sim 5 \times 10^{-10} \mathrm{~cm}$ from Eq. (B2) and, from Eq. (20), the 
corresponding throat radius $a$ is given by

$$
a \sim 0.7 \mathrm{~cm}\left(\frac{d}{5 \times 10^{-10} \mathrm{~cm}}\right)^{3 / 4}\left(\frac{D}{10^{28} \mathrm{~cm}}\right)^{1 / 4}
$$

Therefore, the number density of Ellis wormholes with $a \sim 1 \mathrm{~cm}$ must satisfy $n \lesssim 10^{-9} \mathrm{AU}^{-3}$. Note that this constraint comes from the wave form in the geometrical optics approximation and hence we do not distinguish between the point mass lenses and Ellis wormholes.

Another possible observation to probe Ellis wormholes is the observation of gravitational waves from compact object binaries. The unlensed wave form of the gravitational waves from a compact object binary is well known. From the chirp signal in the inspiral phase, we can obtain the spectrum of the gravitational waves. In order to distinguish the Ellis wormhole from a point mass lens, we need to observe not only the typical interference pattern but also the wave effect in the lensed spectrum. Hence, the spectrum in $d \sim \lambda:=2 \pi / \omega$ is necessary to probe the Ellis wormhole. Assuming $d \sim \lambda$, the typical throat radius of Ellis wormholes which can be probed by using gravitational waves is estimated as follows:

$$
\begin{aligned}
a=d^{3 / 4}\left(\frac{2 D}{\pi^{2}}\right)^{1 / 4} & \sim \lambda^{3 / 4}\left(\frac{2 D}{\pi^{2}}\right)^{1 / 4} \\
& \sim 7 \times 10^{12} \mathrm{~cm}\left(\frac{\lambda}{10^{8} \mathrm{~cm}}\right)^{3 / 4}\left(\frac{D}{10^{28} \mathrm{~cm}}\right)^{1 / 4} .
\end{aligned}
$$

The same estimate is applicable for galactic sources of electro-magnetic waves. We obtain $a \sim 10^{5} \mathrm{~cm}$ for galactic radio sources with $\lambda \sim 1 \mathrm{~cm}, a \sim 1 \mathrm{~m}$ for galactic optical or infra-red sources and $a \sim 1 \mathrm{~cm}$ for galactic X-ray sources. The source must be compact enough to show the clear oscillation behaviour in the spectrum. This fact can be clearly understood by considering the $y$ dependence of the phase in the amplification factor (33). Since $\tau(y)$ is roughly approximated by $2 y$, the period $\delta y$ for one cycle is given by $\delta y \sim \pi /(\omega d)$. The corresponding length scale $\delta \eta$ on the source plane is given by

$$
\begin{aligned}
\delta \eta=\delta y \frac{D_{\mathrm{S}}}{D_{\mathrm{L}}} \xi_{0} & \sim \frac{\pi}{\omega} \sqrt{\frac{2 D_{\mathrm{LS}} D_{\mathrm{S}}}{d D_{\mathrm{L}}}} \\
& \sim \sqrt{\frac{\lambda D_{\mathrm{LS}} D_{\mathrm{S}}}{2 D_{\mathrm{L}}}} \sim 2 \times 10^{11} \mathrm{~cm}\left(\frac{\lambda}{1 \mathrm{~cm}}\right)^{1 / 2}\left(\frac{D_{\mathrm{LS}} D_{\mathrm{S}} / D_{\mathrm{L}}}{10 \mathrm{kpc}}\right)^{1 / 2},
\end{aligned}
$$

where we have assumed $d \sim \lambda$. If the source radius is larger than $\delta \eta$, the interference pattern will be smeared out. Observation of compact galactic sources such as pulsars and white dwarfs might be useful to probe not only dark compact objects but also exotic compact objects such as the Ellis wormhole.

\section{SUMMARY}

In this paper, we have proposed the probe of Ellis wormholes by using spectroscopic observations. We have assumed that the spectrum of the target source can be measured in 
enough accuracy and the spectrum shape is well known without lensing, but the luminosity is not necessarily observable. Then, we have discussed the distinguishability of the lensed spectrum from the case of the point mass lens.

We have derived the wave form after the scattering by the Ellis wormhole including the wave effect in the low frequency domain. The geometrical optics limit of the wave form has been also analytically derived. Then, we have found that the Ellis wormhole cannot be distinguished from the point mass lens by using only the high frequency domain in which the geometrical optics approximation is valid. We have also found that this degeneracy is resolved in the low frequency domain in which the wave effect is significant. Possible observational constraints are also discussed and we estimated the upper bound for the number density of Ellis wormholes as $n \lesssim 10^{-9} \mathrm{AU}^{-3}$ with throat radius $a \sim 1 \mathrm{~cm}$ from the existing femto-lensing analysis for compact objects.

Finally, we note that our method to probe the Ellis wormhole is complementary to the other methods to probe the Ellis wormhole with micro-lensing [21 23] or the astrometric image centroid displacements [26]. These are not feasible for observations on cosmological scales because the time scale of the lens event is too long to detect modulation of the light curve or the displacements. In contrast, the slow relative motion is an advantage for spectroscopic observations. Therefore we may probe the Ellis wormhole on cosmological scales using our method.

\section{Acknowledgements}

CY is supported by a Grant-in-Aid through the Japan Society for the Promotion of Science (JSPS). The work of NT was supported in part by Rikkyo University Special Fund for Research. TH was supported by the Grant-in-Aid for Young Scientists (B) (No. 21740190) and the Grant-in-Aid for Challenging Exploratory Research (No. 23654082) for Scientific Research Fund of the Ministry of Education, Culture, Sports, Science and Technology, Japan.

Appendix A: Derivation of Eq. (14)

The first term in Eq. (13) can be evaluated as follows.

$$
\begin{aligned}
\int_{\mathrm{S}}^{\mathrm{B}} d l=|\overrightarrow{\mathrm{SA}}|+|\overrightarrow{\mathrm{AB}}| & =|\overrightarrow{\mathrm{SA}}|+|\overrightarrow{\mathrm{OA}}-\overrightarrow{\mathrm{OB}}| \\
& =|\overrightarrow{\mathrm{SA}}|+\sqrt{|\overrightarrow{\mathrm{OA}}|^{2}+|\overrightarrow{\mathrm{OB}}|^{2}-2 \overrightarrow{\mathrm{OA}} \cdot \overrightarrow{\mathrm{OB}}}
\end{aligned}
$$

$\overrightarrow{\mathrm{OB}}$ and $\overrightarrow{\mathrm{OB}^{\prime}}$ can be written as

$$
\overrightarrow{\mathrm{OB}}=\left[\left(1-\frac{\delta D}{D_{\mathrm{L}}}\right) \overrightarrow{\mathrm{OL}}+\vec{\eta}+(\vec{\xi}-\vec{\eta}) \frac{D_{\mathrm{LS}}+\delta D}{D_{\mathrm{LS}}}-\alpha \delta D \frac{\vec{\xi}}{\xi}\right]\left(1+\mathcal{O}\left(\epsilon^{3}\right)\right)
$$




$$
\begin{aligned}
& =\left[\left(1-\frac{\delta D}{D_{\mathrm{L}}}\right) \overrightarrow{\mathrm{OL}}+\vec{\xi}-\alpha(\xi) \delta D \frac{\vec{\xi}}{\xi}+(\vec{\xi}-\vec{\eta}) \frac{\delta D}{D_{\mathrm{LS}}}\right]\left(1+\mathcal{O}\left(\epsilon^{3}\right)\right), \\
\overrightarrow{\mathrm{OB}^{\prime}} & =\left(1-\frac{\delta D}{D_{\mathrm{L}}}\right) \overrightarrow{\mathrm{OA}}
\end{aligned}
$$

From these expressions, we can find

$$
\begin{aligned}
|\overrightarrow{\mathrm{OB}}| & =\left|\overrightarrow{\mathrm{OB}^{\prime}}\right|\left(1+\mathcal{O}\left(\epsilon^{3}\right)\right), \\
\overrightarrow{\mathrm{OA}} \cdot \overrightarrow{\mathrm{OB}} & =\overrightarrow{\mathrm{OA}^{\prime}} \cdot \overrightarrow{\mathrm{OB}^{\prime}}\left(1+\mathcal{O}\left(\epsilon^{3}\right)\right) .
\end{aligned}
$$

Therefore we obtain

$$
\int_{\mathrm{S}}^{\mathrm{B}} d l=\left(|\overrightarrow{\mathrm{SA}}|+|\overrightarrow{\mathrm{OA}}|-\left|\overrightarrow{\mathrm{OB}^{\prime}}\right|\right)\left(1+\mathcal{O}\left(\epsilon^{3}\right)\right)
$$

Since we find

$$
\begin{aligned}
|\overrightarrow{\mathrm{SA}}| & =\sqrt{|\vec{\xi}-\vec{\eta}|^{2}+D_{\mathrm{LS}}^{2}}=D_{\mathrm{LS}}\left(1+\frac{|\vec{\xi}-\vec{\eta}|^{2}}{2 D_{\mathrm{LS}}^{2}}+\mathcal{O}\left(\epsilon^{4}\right)\right) \\
|\overrightarrow{\mathrm{AO}}| & =D_{\mathrm{L}}\left(1+\frac{\xi^{2}}{2 D_{\mathrm{L}}^{2}}+\mathcal{O}\left(\epsilon^{4}\right)\right)
\end{aligned}
$$

we obtain the following expression:

$$
\int_{\mathrm{S}}^{\mathrm{B}} d l=\left[D_{\mathrm{S}}\left(1+\frac{\eta^{2}}{2 D_{\mathrm{S}}^{2}}\right)+\frac{D_{\mathrm{L}} D_{\mathrm{S}}}{2 D_{\mathrm{LS}}}\left(\frac{\vec{\xi}}{D_{\mathrm{L}}}-\frac{\vec{\eta}}{D_{\mathrm{S}}}\right)^{2}-r\right]\left(1+\mathcal{O}\left(\epsilon^{3}\right)\right)
$$

where $r=\left|\overrightarrow{\mathrm{OB}^{\prime}}\right|$.

In order to evaluate the second term in Eq. (13), we first consider the integral between $\mathrm{S}$ and A. Letting $\mathrm{P}$ be a point on the segment $\mathrm{SA}$, we obtain

$$
|\overrightarrow{\mathrm{LP}}|^{2}=|\vec{\xi}+\overrightarrow{\mathrm{AP}}|^{2}=\left|\vec{\xi}+\left(1-\frac{l}{l_{\mathrm{SA}}}\right) \overrightarrow{\mathrm{AS}}\right|^{2}=\xi^{2}+\left(l_{\mathrm{SA}}-l\right)^{2}+2\left(1-\frac{l}{l_{\mathrm{SA}}}\right) \vec{\xi} \cdot \overrightarrow{\mathrm{AS}}
$$

where $l_{\mathrm{SA}}=|\overrightarrow{\mathrm{SA}}|$ and $l=|\overrightarrow{\mathrm{SP}}|$. Since $\vec{\xi} \cdot \overrightarrow{\mathrm{AS}}=-\vec{\xi} \cdot(\vec{\xi}-\vec{\eta})$, we obtain the following expression:

$$
|\overrightarrow{\mathrm{LP}}|^{2}=\xi^{2}+\left(l_{\mathrm{SA}}-l\right)^{2}-2\left(1-\frac{l}{l_{\mathrm{SA}}}\right) \vec{\xi} \cdot(\vec{\xi}-\vec{\eta}) \text {. }
$$

Substituting the above expression of $|\overrightarrow{\mathrm{LP}}|^{2}$ into $R^{2}$ of the second integral in Eq. (13) with the integral region being from $\mathrm{S}$ to $\mathrm{A}$, we obtain

$$
\int_{\mathrm{S}}^{\mathrm{A}} \frac{1}{R^{2}} d l=\int_{0}^{l_{\mathrm{SA}}} \frac{d l}{\xi^{2}+\left(l_{\mathrm{SA}}-l\right)^{2}-2\left(1-\frac{l}{l_{\mathrm{SA}}}\right) \vec{\xi} \cdot(\vec{\xi}-\vec{\eta})}
$$


Then the integral (A12) can be performed and evaluated as

$$
\begin{aligned}
& \int_{0}^{l_{\mathrm{SA}}} \frac{d l}{\left(l_{\mathrm{SA}}-l\right)^{2}+\xi^{2}-2\left(1-\frac{l}{l_{\mathrm{SA}}}\right) \vec{\xi} \cdot(\vec{\xi}-\vec{\eta})}=\frac{l_{\mathrm{SA}}}{\xi D_{\mathrm{LS}}}\left[\arctan \left(\frac{-l_{\mathrm{SA}}^{2}+\vec{\xi} \cdot(\vec{\xi}-\vec{\eta})+l l_{\mathrm{SA}}}{\xi D_{\mathrm{LS}}}\right)\right]_{0}^{l_{\mathrm{SA}}} \\
& =\left(\frac{\pi}{2 \xi}-\frac{\vec{\xi} \cdot \vec{\eta}}{\xi^{2} D_{\mathrm{LS}}}\right)\left(1+\mathcal{O}\left(\epsilon^{2}\right)\right) \text {. }
\end{aligned}
$$

The contribution from the integral between A and B can be also evaluated by the similar integral. Finally, we obtain the expression (14).

\section{Appendix B: Point Mass Lens}

For the point mass case, we have the following expression for the amplification factor [28, 29]:

$$
\left|F^{\mathrm{po}}\right|=\left|\mathrm{e}^{\pi \omega d / 2} \Gamma(1-i \omega d)_{1} F_{1}(i \omega d, 1 ; i \omega d y)\right|
$$

where $\Gamma$ and ${ }_{1} F_{1}$ are the gamma function and the confluent hyper-geometric function, respectively, and

$$
d=2 M, \quad y=\eta \sqrt{\frac{D_{\mathrm{L}}}{4 M D_{\mathrm{LS}} D_{\mathrm{S}}}} .
$$

In the geometrical optics approximation $(\omega \rightarrow \infty)$, we obtain

$$
\left|F^{\mathrm{po}}\right|^{2} \rightarrow\left|F_{\mathrm{geo}}^{\mathrm{po}}\right|^{2}:=\left|\mu_{+}^{\mathrm{po}}\right|+\left|\mu_{-}^{\mathrm{po}}\right|+2 \sqrt{\left|\mu_{+}^{\mathrm{po}} \mu_{-}^{\mathrm{po}}\right|} \sin \left(2 \omega d \tau_{\mathrm{po}}(y)\right),
$$

where

$$
\begin{aligned}
\mu_{ \pm}^{\mathrm{po}} & = \pm \frac{1}{4}\left[\frac{y}{\sqrt{y^{2}+4}}+\frac{\sqrt{y^{2}+4}}{y} \pm 2\right], \\
\tau_{\mathrm{po}}(y) & =\frac{1}{2} y \sqrt{y^{2}+4}+\ln \frac{\sqrt{y^{2}+4}+y}{\sqrt{y^{2}+4}-y} .
\end{aligned}
$$

$\left|F^{\mathrm{po}}\right|^{2}$ and $\left|F_{\text {geo }}^{\mathrm{po}}\right|^{2}$ are depicted as functions of $\omega$ for each value of $y$ in Fig. 8 ,

[1] MACHO Collaboration, C. Alcock et al., Astrophys.J. 542, 281 (2000), arXiv:astro-ph/0001272, The MACHO project: Microlensing results from 5.7 years of LMC observations.

[2] EROS-2 Collaboration, P. Tisserand et al., Astron.Astrophys. 469, 387 (2007), arXiv:astro-ph/0607207, Limits on the Macho Content of the Galactic Halo from the EROS-2 Survey of the Magellanic Clouds. 

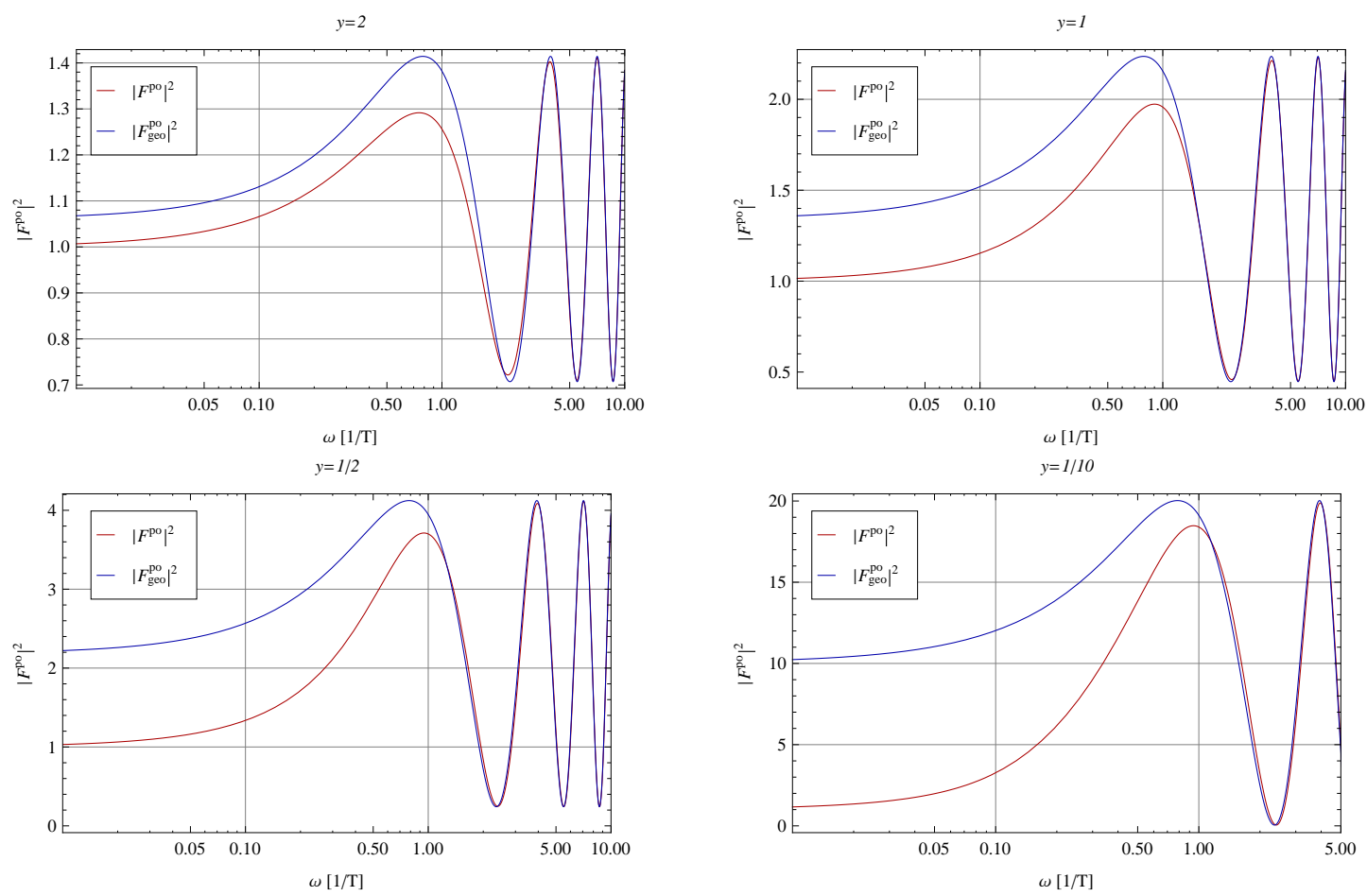

FIG. 8: $\left|F^{\mathrm{po}}\right|^{2}$ and $\left|F_{\text {geo }}^{\mathrm{po}}\right|^{2}$ as functions of $\omega$ for each value of $y$, where $T=d \tau_{\text {po. }}$.

[3] L. Wyrzykowski et al., Mon.Not.Roy.Astron.Soc. 397, 1228 (2009), arXiv:0905.2044, The OGLE View of Microlensing towards the Magellanic Clouds. I. A Trickle of Events in the OGLE-II LMC data.

[4] D. Huterer and T. Vachaspati, Phys.Rev. D68, 041301 (2003), arXiv:astro-ph/0305006, Gravitational lensing by cosmic strings in the era of wide - field surveys.

[5] M. Oguri and K. Takahashi, Phys.Rev. D72, 085013 (2005), arXiv:astro-ph/0509187, Characterizing a cosmic string with the statistics of string lensing.

[6] K. J. Mack, D. H. Wesley, and L. J. King, Phys.Rev. D76, 123515 (2007), arXiv:astro-ph/0702648, Observing cosmic string loops with gravitational lensing surveys.

[7] K. Kuijken, X. Siemens, and T. Vachaspati, MNRAS384, 161 (2008), arXiv:0707.2971, Microlensing by cosmic strings.

[8] J. Christiansen et al., Phys.Rev. D83, 122004 (2011), arXiv:1008.0426, Search for Cosmic Strings in the COSMOS Survey.

[9] A. Tuntsov and M. Pshirkov, Phys.Rev. D81, 063523 (2010), arXiv:1001.4580, Quasar variability limits on cosmological density of cosmic strings.

[10] M. Pshirkov and A. Tuntsov, Phys.Rev. D81, 083519 (2010), arXiv:0911.4955, Local con- 
straints on cosmic string loops from photometry and pulsar timing.

[11] D. Yamauchi, K. Takahashi, Y. Sendouda, and C.-M. Yoo, Phys.Rev. D85, 103515 (2012), arXiv:1110.0556, Weak lensing of CMB by cosmic (super-)strings.

[12] D. Yamauchi, T. Namikawa, and A. Taruya, JCAP 1210, 030 (2012), arXiv:1205.2139, Weak lensing generated by vector perturbations and detectability of cosmic strings.

[13] H. Ellis, J.Math.Phys. 14, 104 (1973), Ether flow through a drainhole - a particle model in general relativity.

[14] H.-a. Shinkai and S. A. Hayward, Phys.Rev. D66, 044005 (2002), arXiv:gr-qc/0205041, Fate of the first traversible wormhole: Black hole collapse or inflationary expansion.

[15] A. Das and S. Kar, Class.Quant.Grav. 22, 3045 (2005), arXiv:gr-qc/0505124, The Ellis wormhole with 'tachyon matter'.

[16] L. Chetouani and G. Clement, General Relativity and Gravitation 16, 111 (1984), Geometrical optics in the Ellis geometry.

[17] G. Clément, International Journal of Theoretical Physics 23, 335 (1984), Scattering of KleinGordon and Maxwell Waves by an Ellis Geometry.

[18] K. Nakajima and H. Asada, Phys.Rev. D85, 107501 (2012), arXiv:1204.3710, Deflection angle of light in an Ellis wormhole geometry.

[19] N. Tsukamoto and T. Harada, (2012), arXiv:1211.0380, Signed magnification sums for general spherical lenses.

[20] D. F. Torres, G. E. Romero, and L. A. Anchordoqui, Phys.Rev. D58, 123001 (1998), arXiv:astro-ph/9802106, Might some gamma-ray bursts be an observable signature of natural wormholes?

[21] M. Safonova, D. F. Torres, and G. E. Romero, Phys.Rev. D65, 023001 (2002), arXiv:gr-qc/0105070, Microlensing by natural wormholes: Theory and simulations.

[22] M. Bogdanov and A. Cherepashchuk, Astrophys.Space Sci. 317, 181 (2008), arXiv:0807.2774, Search for exotic matter from gravitational microlensing observations of stars.

[23] F. Abe, Astrophys.J. 725, 787 (2010), arXiv:1009.6084, Gravitational Microlensing by the Ellis Wormhole.

[24] H. Asada, Prog.Theor.Phys. 125, 403 (2011), arXiv:1101.0864, Gravitational microlensing in modified gravity theories: Inverse-square theorem.

[25] T. Kitamura, K. Nakajima, and H. Asada, (2012), arXiv:1211.0379, Demagnifying gravita- 
tional lenses toward hunting a clue of exotic matter and energy.

[26] Y. Toki, T. Kitamura, H. Asada, and F. Abe, Astrophys.J. 740, 121 (2011), arXiv:1107.5374, Astrometric Image Centroid Displacements due to Gravitational Microlensing by the Ellis Wormhole.

[27] N. Tsukamoto, T. Harada, and K. Yajima, (2012), arXiv:1207.0047, Can we distinguish between black holes and wormholes by their Einstein ring systems?

[28] S. Deguchi and W. D. Watson, Phys.Rev. D34, 1708 (1986), Wave effects in gravitational lensing of electromagnetic radiation.

[29] P. Schneider, J. Ehlers, and E. E. Falco, Gravitational Lenses (, 1992).

[30] C. Baraldo, A. Hosoya, and T. T. Nakamura, Phys. Rev. D59, 083001 (1999), Gravitationally induced interference of gravitational waves by a rotating massive object.

[31] A. Mehrabi and S. Rahvar, arXiv:1207.4034 [astro-ph.EP].

[32] R. Takahashi and T. Nakamura, Astrophys. J. 595, 1039 (2003), astro-ph/0305055, Wave effects in gravitational lensing of gravitational waves from chirping binaries.

[33] T. Suyama, T. Tanaka, and R. Takahashi, Phys.Rev. D73, 024026 (2006), arXiv:astro-ph/0512089, Exact wave propagation in a spacetime with a cosmic string.

[34] C.-M. Yoo, R. Saito, Y. Sendouda, K. Takahashi, and D. Yamauchi, (2012), arXiv:1209.0903, Femto-lensing due to a Cosmic String.

[35] M. Born and E. Wolf, Principles of Optics (, 1999).

[36] A. Gould, ApJ386, L5 (1992), Femtolensing of gamma-ray bursters.

[37] K. Z. Stanek, B. Paczynski, and J. Goodman, ApJ413, L7 (1993), Features in the spectra of gamma-ray bursts.

[38] A. Ulmer and J. Goodman, Astrophys.J. 442, 67 (1995), arXiv:astro-ph/9406042, Femtolensing: Beyond the semiclassical approximation.

[39] G. Marani, R. Nemiroff, J. Norris, K. Hurley, and J. Bonnell, (1998), arXiv:astro-ph/9810391, Gravitationally lensed gamma-ray bursts as probes of dark compact objects.

[40] A. Barnacka, J. Glicenstein, and R. Moderski, Phys.Rev. D86, 043001 (2012), arXiv:1204.2056, New constraints on primordial black holes abundance from femtolensing of gamma-ray bursts. 